\title{
Scattering of $x$ rays from low-Z materials
}

J. L. Gaines
L. D. Kissel
H. C. Catron
R. A. Hansen

Mrop?

A ugust 1, 1980

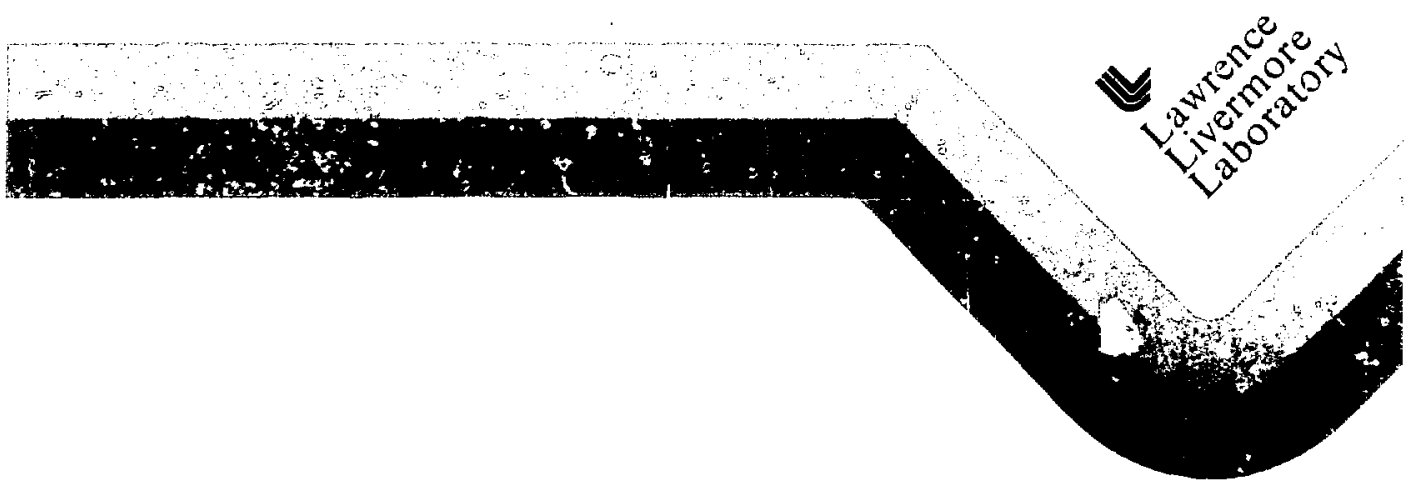


I 'CRI.-52968

Distribution Catcgory I ( -25

\title{
Scattering of $x$ rays from low-Z materials
}

\author{
J. L. Gaines \\ L. D. Kissel \\ H. C. Catron \\ R. A. Hansen
}

Manuscript date: August 1, 1980

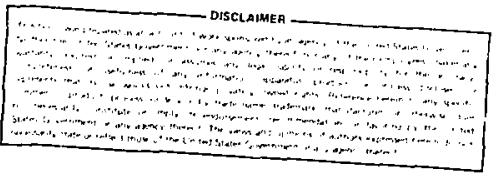

\section{LAWRENCE LIVERMORE LABORATORY}

University of California $\bullet$ Livermore, California 94550 


\section{CONTENTS}

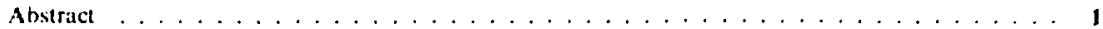

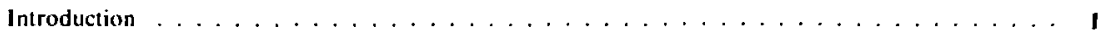

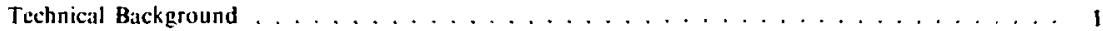

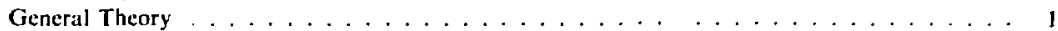

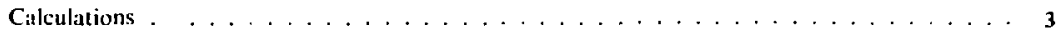

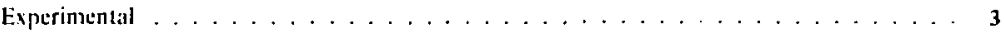

Theortical ...........................

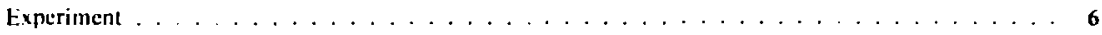

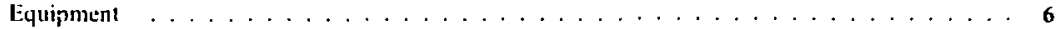

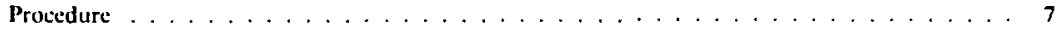

Resulss and Diseussion $\ldots \ldots \ldots \ldots \ldots \ldots \ldots \ldots \ldots$

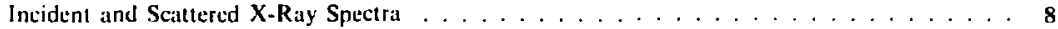

Relative Scattering Efficiency . . . . . . . . . . . . . . . . . 9

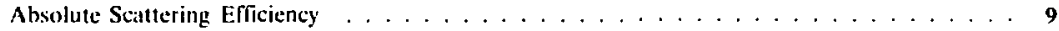

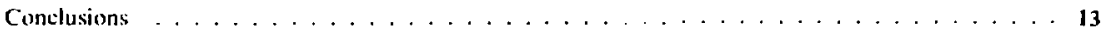

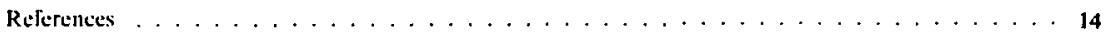




\section{Scattering of $x$ rays \\ from low-Z materials}

\section{ABSTRACT}

$X$ rays incident on thin beryllium, boron, carbon, and other low- $Z$ materials undergo both elastic and inelastic scattering as well as diffraction from the crystalline or "crystalline-like" structure of the material. Unpolarized monoenergetic $x$ rays in the 1.5- to $8.0-\mathrm{keV}$ energy range were used to deterınine the absolute scattering efficiency of thin beryllium, carbon, and boron foils. These measurements are compared to calculated scattering efficiencies predicted by single-atom theories. In addition, the relative scattering efficiency versus $x$-ray energy was measured for other low- $Z$ foils using unpolarized brensstrahlung $x$ rays. In all the low- $Z$ foils examined, we observed Bragg-like $x$-ray diffraction due to the ordered structure of the materials.

\section{INTRODUCTION}

Low- $Z$ materials are often used or considered for use as scatterers of $x$-ray beams. These uses may originate from a desire to reduce the intensity of the $x$-ray beam. e.g.. for diagnostic purposes, or may be required as a result of experimental geometry constraints. It is necessary to know the energy distribution and intensity of the $x$ rays scattered from the low- $Z$ material in order to make effective use of it as a scatterer.

Although a considerable amount of work has been done in the area of calculating or predicting single-atom photon scattering, ${ }^{1,2}$ little experimental work has been performed in the 1- to 10-keV energy range to confirm these calculations and predictions.
We have therefore measured the absolute seattering efficiency in this energy range of three thin low- $Z$ foils ( $B e, C$, and $B)^{3}$ and compared these values with calculated $\sin _{0}$ le-atom scattering efficiencies. These measurements were made using intense, nearly monocnergetic, unpolarized $x$-ray beams in the

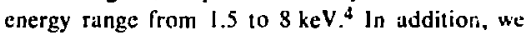
have measured the relative scattering efficiency of several other thin, low- $Z$ foils using unpolarized 16keV bremsstrahlung $x$ rays from $\mathrm{Al}$ and $\mathrm{Cu}$ targets. High resolution lithium-drifted silicon detectors were used to monitor the incident and scattered $x$ rays.

\section{TECHNICAL BACKGROUND}

\section{GENERAL THEORY}

In traversing a thin slab of matter, a narrow beam of $x$ rays is absorbed in accordance with the usual exponential equation

$$
I=I_{0} e^{-\mu x},
$$

where $J$ is the transmitted intensity, and $I_{0}$ is the incident intensity of the $x$-ray beam. The quantity $x$ is the thickness of the material in centimeters, and $\mu$ is the linear absorption coefficient. We may rewrite this equation as

$$
I=I_{0} e^{-(\mu / \rho) x},
$$

where $\rho$ is the material density. The quantity $\mu / \rho$, the mass absorption coefficient, is more nearly independent of the physical and chemical state of the material than $\mu$. The loss of intensity of a narrow $x$-ray beam as it traverses the material is actually due to two processes, "true absorption" and "scatter." True absorption involves the transformation 
of $x$ says into kinetic energy of ejected electrons, whereas scattering is a process in which radiant energy is transferred from the primary $x$-ray beam to scattered beams originating in the atoms of the absorber. The mass absorption coefficient can thus be written as

$$
\frac{\mu}{\rho}=(\tau / \rho)+(\sigma / \rho)
$$

where $\tau / \rho$ is the true absorption coefficient, and $\sigma / \rho$ is the scattering coefficient. Although this true absorption coefficient accounts for most of the loss of intensity as $x$ rays traverse a substance, in this paper we are primarily concerned with the smaller fraction of beam intensity lost through scutlering.

Scattering can be classified as either elastic or inelastic. Elisstic scattering involves a change in $x$-ray momentum but no change in $x$-ray energy, i.e., a change of direction only. A portion of the elastically scattcred radiation comprises the phenomenon of $x$-ray diffraction, or "Bragg scattering," which will be discussed in more detail later. Inelastic scattering involves both a change in $x$-ray momcntum and $x$-ray energy. Thus we can write the total scattering cross section for an isolatcd atom as the sum of the elastic and inelastic scattering cross sections,

$$
\sigma_{\text {scat }}=\sigma_{\text {elas }}+\sigma_{\text {inelas }} \text {. }
$$

If we consider only scattering which is observed in a small solid angle $d \Omega$ at a specified angle $\theta$ with respect to the incident $x$-ray beam, then we can define the differential scattering cross section per unit solid angle as

$$
\frac{d \sigma_{\text {scat }}}{d \Omega}=\frac{d \sigma_{\text {elas }}}{d \Omega}+\frac{d \sigma_{\text {inelas }}}{d \Omega} .
$$

In principle, it is impossible to distinguish which component of an isolated atom is responsible for elastically scattering a photon since there is no change in the atom after scattering. However, in practice, one usually expresses the total-atom elastic scattering amplitude, for scattering $x$ rays and $\gamma$ rays, as a sum of amplitudes due to elastic scattering by the: ing).

(I) Bound atomic electrons (Rayleigh scatter-

(2) Nuclear charge (nuclear Thomson scattering).

(3) Structure of the nucleus (nuclear resonance scattering).

(4) Field of the nucleus (Delbriick scattering). The total-atom elastic scattering cross section is the square of the total scattering amplitude. For x-ray energies above $100 \mathrm{keV}$, all of these processes are important. However, the energies of the $x$ rays used in this experiment were in the range 1 to $20 \mathrm{keV}$. and in this energy region Rayleigh scattering strongly dominates the total-atom elastic scattering cross section. The differential cross section for Rayleigh scattering per unit solid angle is often written in terms of a form-factor-corrected Thomson cross section, ${ }^{5}$

$$
\frac{\mathrm{d} \sigma_{\text {clas }}}{d \Omega}=|F(q)|^{2} \frac{d \sigma_{\text {Thomson }}}{d \Omega} .
$$

The form factor $F(q)$ corrects the point-charge Thomson scattering cross section for scattering by an extended charge distribution. The Thomson cross section is given by ${ }^{5}$

$$
\frac{\mathrm{d} \sigma_{\text {Thomson }}}{\mathrm{d} \Omega}=\frac{\mathrm{r}_{0}{ }^{2}}{2}\left(1+\cos ^{2} \theta\right),
$$

where $\theta$ is the scattering angle and $\mathbf{r}_{0}$ is the classical radius of the electron given as

$$
I_{0}=\frac{e^{2}}{m c^{2}} \text {. }
$$

with

$$
\begin{aligned}
& \mathrm{e}=\text { electron charge. } \\
& \mathrm{m}=\text { electron rest mass. }
\end{aligned}
$$$$
c=\text { velocity of light. }
$$

The atomic form factor $F(q)$ is given for a spherically symmetric electronic charge density by ${ }^{5}$

$$
F(q)=4 \pi \int_{0}^{\infty} \rho(r) \frac{\sin (q r)}{q r} r^{2} d r,
$$

where

$\rho(r)=$ radial electron charge density,

$r=$ atomic radial coordinate centered on the nucleus,

$4=4 \pi / \lambda \sin \theta / 2(\theta=$ scattering angle and $\lambda=$ phot on wavelength). 
For $\mathrm{x}$ tays with $\mathrm{E}<20 \mathrm{keV}$, the inelastic scattering by atoms is due primarily to the contribution made by the atomic electrons and is referred to as bound Compton scattering. The inelastic scattering from an atom containing $N$ bound electrons is often calculated using a scattering function correction to the Klein-Nishina formula ${ }^{6}$ :

$$
\frac{d \sigma_{\text {inelas }}}{d \Omega}=S\left(q_{\text {incoh }}\right) \frac{d \sigma_{K N}}{d \Omega},
$$

vhere

$$
\begin{aligned}
h \mathrm{~g}_{\text {incoh }}= & 2 \mathrm{~K} \sin 0 / 2\left[1+\left(\mathrm{K}^{2}+2 \mathrm{~K}\right)\right. \\
& \left.\times \sin ^{2}(\theta / 2)\right]^{1 / 2} /\left[1+2 \mathrm{~K} \sin ^{2}(\theta / 2)\right] .
\end{aligned}
$$

and the Klein-Nishina formula may be written as

$$
\begin{aligned}
& \frac{d \sigma_{\mathrm{KN}}}{\mathrm{d} \Omega}=\frac{\mathrm{r}_{0}^{2}\left(1+\cos ^{2} \theta\right)}{2[1+\mathrm{k}(1-\cos \theta)]^{2}} \\
& \quad \times\left\{1+\frac{\mathrm{k}^{2}(1-\cos \theta)^{2}}{\left[1+\cos ^{2} \theta\right][1+k(1-\cos \theta)]}\right\},
\end{aligned}
$$

where

$$
\begin{aligned}
& k=h \nu_{0} / \mathrm{mc}^{2} \text { energy of incident } \mathrm{x} \text { ray. } \\
& \mathrm{r}_{0}=\text { classical radius of electron as given in }
\end{aligned}
$$
Eq. (8).

$\theta=\mathrm{x}$-ray scattering angle.

As with elastic scattering by atomic electrons, the incoherent scattering function $S$ ( $q_{\text {incoh }}$ ) corrects the free point charge Klein-Nishina formula for scattering by a bound and distributed electronic charge. Tables have been published which give caiculated values for the incoherent scattering functions. ${ }^{1}$

The phenomenon of $x$-ray diffraction by crystals results from elastic scattering by a collection of atoms which are fixed it. a spatial lattice. Diffraction occurs only when certain geometrical conditions are satisfied, which are expressed for crystals in terms of the Bragg condition?:

$$
\mathrm{n} \lambda=2 \mathrm{~d} \sin \theta_{\beta},
$$

where

$\mathbf{n}=$ order of reflection,

$\lambda=$ wavelength in $A$ of incident $x$ ray.

$\mathrm{d}=$ interplanar spacing in $A$,

$\theta_{i}=$ angle between the atomic plane and the incident beam.

For well-ordered crystals with known d spacings this relationship is routinely used in most applications of $\mathrm{x}$-ray diffraction systems. However, diffraction is not limited to well-ordered crystalline substances but occurs from most solids when the right geometric conditions are present. It is now evident that most materials act as three-dimensional diffraction gratings for $x$ rays in the $1-$ to $20-\mathrm{keV}$ energy range. In fact, it appears that a truly amorphous scatterer is an extreme rarity for $x$ lays in this energy rangc.

Considering all the processes which contribute to the scattering of $x$ rays from materials in the energy region under consideration. Eq. (5) should be modified to include a contribution due to diffrac:ion. Howsver, at the present time we are unable to properly quantify the contribution due to this effect.

\section{CALCULATIONS}

\section{Experimental}

In this experiment we have measured the absolute scattering efficiency which we have defined as the fraction of $x$ rays of energy $E$ incident on the scatterer and scattered into a solid angle $\Omega$.

$$
\begin{aligned}
& E(E, \Omega) \\
& =\frac{\text { No. scattered } x \text { rays into solid angle } \Omega}{\text { No. } x \text { rays of energy } E \text { incicient }} .
\end{aligned}
$$

Figure I illustrates the typical geometry used in this experiment. Referring to this figure, we see that all of the $x$ rays that pass through collimator $C_{1}$ strike the scattering foil, and all of the $x$ rays that pass through $C_{2}$ strike detector No. 1 which is a windowless lithium-drifted silicon detector. The number of $x$ rays per second striking the foil are rejated to the number of $x$ rays counted by detector No. I by the relation

$$
\dot{\mathrm{N}}_{\mathrm{C}_{1}}=\frac{\mathrm{N}_{\mathrm{C}_{2}}}{\mathrm{te}_{1}}\left(\frac{\mathrm{D}_{\mathrm{C}_{1}}}{\mathrm{D}_{\mathrm{C}_{2}}}\right)^{2}\left(\frac{\mathrm{R}_{\mathrm{C}_{2}}}{\mathrm{R}_{\mathrm{C}_{1}}}\right)^{2},
$$




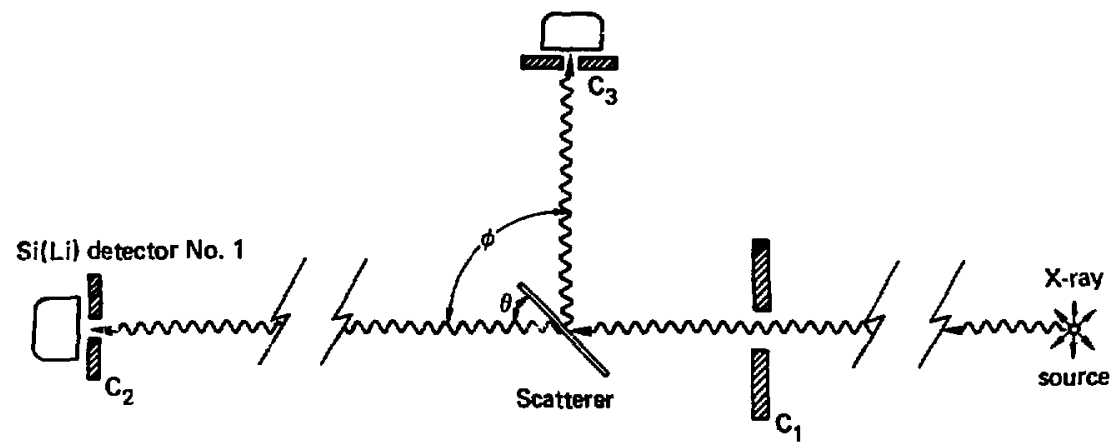

FIG. 1. Experimental geometry.

where

$\dot{\mathrm{N}}_{\mathrm{Cl}}=\mathrm{x}$ rays per second striking the foil,

$\mathrm{D}_{\mathrm{Cl}_{1}}=$ diameter of collimator $\mathrm{C}_{1}$ in $\mathrm{cm}$,

$R_{C_{1}}=$ distance from $x$-ray source to $C_{1}$ in $\mathrm{cm}$,

$D_{C_{2}}=$ diameter of collimator $C_{2}$ in $\mathrm{cm}$,

$R_{\mathrm{C}_{2}}=$ distance from $x$-ray source to $\mathrm{C}_{2}$ in $\mathrm{cm}$, time $\mathrm{t}$,

$\mathrm{N}_{\mathrm{C}_{2}}=\mathrm{x}$ rays counted by detector No. 1 in

$1=$ counting interval in seconds,

$e_{1}=$ efficiency of detector No. 1 .

All of the $x$ rays that are scattered from the foil and pass through collimator $C_{3}$ of Fig. 1 are counted by detector No. 2. The solid angle subtended by $\mathrm{C}_{3}$ is given by

$$
\Omega=\frac{\text { Area of collimator } C_{3}}{\text { Distance from scatterer to } C_{3}}
$$

$$
=\frac{(\pi / 4)\left(D_{C_{3}}\right)^{2}}{R_{C_{3}}}
$$

where

$\mathrm{D}_{\mathrm{C}_{3}}=$ diameter of collimator $\mathrm{C}_{3}$ in $\mathrm{cm}$,

$\mathbf{R}_{\mathrm{C}_{3}}=$ distance from scatterer $\mathrm{to}_{3}$ in $\mathrm{cm}$.

Referring again to Fig. 1, the number of $x$ rays per second scattered through collimator $C_{3}$ and counted by detector No. 2, a lithium-drifted silicon detector with a $5.08 \times 10^{-3}$-cm-thick beryllium window, is given by

$$
\dot{N}_{C_{3}}=\frac{N_{C_{3}}}{t_{2} e_{2}}
$$

where

$\mathrm{N}_{\mathrm{C}_{3}}=$ number of $\mathrm{x}$ rays counted by detector No. 2 in $t_{2}$ seconds,

$t_{2}=$ counting interval in seconds,

$\mathrm{e}_{2}=$ efficiency of detector No. 2.

Thus the number of $x$ rays per second per steradian scattered into detector No. 2 is

$$
\dot{\mathrm{N}}_{\mathrm{S}_{3}}=\frac{\dot{\mathrm{N}}_{\mathrm{C}_{3}}}{\Omega},
$$

and the measured scattering efficiency is then

$$
\epsilon(E, \Omega)=\frac{\dot{\mathrm{N}}_{\mathrm{S}_{3}}}{\overline{\mathrm{N}}_{\mathrm{C}_{1}}} .
$$

\section{Theoretical}

Kissel ${ }^{8}$ relates single-atom elastic and inelastic scattering cross sections to the experimentally observed scattering efficiency for thin foils. We repeat expression 13 from this reference here as

$$
\epsilon(E, \Omega)=n t_{\text {eles }} \frac{d \sigma_{\text {elus }}}{d \Omega}+n t_{\text {inelss }} \frac{d \sigma_{\text {inelas }}}{d \Omega}
$$


where $\mathrm{cm}^{3}$

$\mathrm{n}=$ scatterer number density in scatterers/

$t_{\text {elas }}=$ effective thickness of material perpendicular to the incident $x$-ray beam direction for elastic scattering,

$\mathrm{d} \sigma_{\text {elas }} / \mathrm{d} \Omega=$ differential clastic scattering cross section per scatterer,

$t_{\text {inelas }}=$ effective thickness of material perpendicular to the incident $x$-ray beam direction for inelastic scattering,

$\mathrm{d} \sigma_{\text {inelas }} / \mathrm{d} \Omega=$ differential inelastic scattering cross section per scatterer.

The quantities $t_{e l a s}$ and tinelas are effective thicknesses of material in the $x$-ray beam. That is, $\mathrm{nt}_{\text {elas }}$ is the number of scatterers per unit area of incident $x$-ray beam which effectively contribute to elastic scattering.

For a reflection geometry $\theta>\phi$, the $x$-ray source and detectors are on the same side of the scattering foil. The effective foil thicknesses are:

$$
=\frac{1-\exp \left\{-n\left[\frac{\sigma(E)}{\sin \phi}+\frac{\sigma(E)}{\sin (\theta-\phi)}\right] t\right\}}{n\left[\sigma(E)+\frac{\sigma(E) \sin \phi}{\sin (\theta-\phi)}\right]},
$$

and

$t \stackrel{\text { ref }}{\text { inelas }}$

$$
=\frac{1-\exp \left\{-n\left[\frac{\sigma(E)}{\sin \phi}+\frac{\sigma\left(E^{\prime}\right)}{\sin (\theta-\phi)}\right] t\right\}}{n\left[\sigma(E)+\frac{\sigma\left(E^{\prime}\right) \sin \phi}{\sin (\theta-\phi)}\right]} ;
$$

where

$E=$ energy of incident $x$ ray and energy of elastically scattered $x$ ray,

$E^{\prime}=$ energy of inelastically scattered $x$ ray, $\sigma(\omega)=$ total cross section for photons of energy $\omega$,

$\mathrm{t}=$ physical foil thickness,

$\theta=$ scattering angle,

$\phi=$ foil angle.

In a transmission geometry, $\phi>\theta$, the $x$-ray source and detector are on opposite sides of the scattering foil. The effective thicknesses are:

$$
\begin{aligned}
\mathrm{t}_{\text {eles }}^{\mathrm{Cusn}} & =\exp -\frac{\mathrm{no}(E) \mathrm{t}}{\sin \phi} \\
\times & \frac{1-\exp \left\{n\left[\frac{\sigma(E)}{\sin \phi}-\frac{\sigma(E)}{\sin (\phi-\theta)}\right] \mathrm{t}\right\}}{\mathrm{n}\left[\frac{\sigma(E) \sin \phi}{\sin (\phi-\theta)}-\sigma(E)\right]},
\end{aligned}
$$

and

$$
\begin{aligned}
\mathbf{t}_{\text {inelas }}^{\text {trus }} & =\exp -\frac{n \sigma(E) t}{\sin \phi} \\
\times & \frac{1-\exp \left\{n\left[\frac{\sigma(E)}{\sin \phi}-\frac{\sigma\left(E^{\prime}\right)}{\sin (\phi-\theta)}\right] t\right\}}{n\left[\frac{\sigma\left(E^{\prime}\right) \sin \phi}{\sin (\phi-\theta)}-\sigma(E)\right]} .
\end{aligned}
$$

(See Fig. $I$ for definition of $\theta$ and $\phi$.)

Kissel has written a code called SEFF which calculates the scattering efficiency of Eq. (19) for fixed $\theta$ at various $E$. This code uses $E q$. (6) which includes the form-factor-corrected Thomson formula to calculate the contribution from elastic scattering and which uses Eq. (10), the incoherent-scatteringfunction-corrected Klein-Nishina formula, to calculate the contribution from inelastic scattering. The code obtains the required values of $F(q)$ and $S(q)$ from a private library containing the values published by Hubbell et al. ' The values of $F(q)$ and $S(q)$ for $Z=2-6$ were derived from nonrelativistic configuration-interaction calculations which include substantial electron correlation effects. The total cross sections required for the calculation of the effective thicknesses, Eqs. (20) to (23), were obtained from the L Division cross-section library CSLOW3/77.

Precise limitations on the accuracy of the formfactor-corrected Thomson formula, Eq. (6), are not presently available. Recent investigations ${ }^{2,10,11}$ do provide general guidance on regions of expected validity for Eq. (6). It is expected that Eq. (6) will be valid for light elements $(Z \leqq 20)$, for energies well above $K$ shell binding, and for values of the momentum transfer $q<20 A^{-1}$. That is, Eq. (6) should be generally valid as used here. General limits on the validity of $S(q)$ are not known. 


\section{EXPERIMENT}

\section{EQUIPMENT}

The $x$-ray facility used to provide the monoinergetic $x$ rays in the 1.5- $108-\mathrm{keV}$ energy range as well as the bremsstrahlung $x$-ray beams is a part of the Lawrence Livermore National Laboratory $X$-Ray Calibration and Stanoards Laboratory 12 and has been described in detail previous! $:{ }^{4}$ The foils which were examined are listed in Table 1 below.

The foils or scatterers were $>0.99$ pure in most cases. An oxide coating was present on the beryllium and aluminum as no effort was made to reduce the effects of oxidation, and care was taken only to protect them from physical damage. The foils were placed in a vacuum of $\leqslant 10^{-6}$ Torr for more than one hour before being used in the experiment.

The thicknesses given are either those specified by the manufacturer or were determined by measuring the mass per unit area through precision weighing. The two beryllium foils were made by differing processes. One was vapor plated $(1.16 \times$ $\left.10^{-3} \mathrm{~g} / \mathrm{cm}^{2}\right)$, and the other was rolled to the proper thickness $\left(1.39 \times 10^{-3} \mathrm{~g} / \mathrm{cm}^{2}\right)$. The vapor plated foil should be of higher purity because impurities can be introduced by the rolling process. The boron and carbon foils were fabricated by depositing boron and carbon on thin polyethylene or parylene foils. ${ }^{3}$ In the case of the boron and carbon on parylene, the parylene foils were measured for their relative scattering efficiency before the boron or carbon was deposited, anc then the scattering efficiency for the coated foils was measured. This allowed us to determine the effect of the substrate.

The two liquid-nitrogen-cooled, lithium-drifted silicon detectors were very similar with the exception that one detector had a $5.08 \times 10^{-3}-\mathrm{cm}$-thick beryllium window, and the other one was used in a windowless mode. These detectors are $100 \%$ absorbing for $16-\mathrm{keV} x$ rays and have an energy resolution of $185 \mathrm{eV}$ for the ${ }^{55} \mathrm{Fe}$ radiation lines.

The scattering foils were mounted on frames attached to a positioning mechanism that would allow adjustment from outside of the evacuated scattering chamber. The positioning mechanism could hold and position three foils with an empty fourth position to allow the beam a straightthrough path. This apparatus can be seen in Fig. 2 .

The data was recorded using a multichannel pulse height analyzer system as well as typical counting electronics; and stored on magnetic tape and processed using the Lawrence Livermore National Laboratory LTSS computer facility. ${ }^{13}$

TABLE 1. List of scatterers

\begin{tabular}{|c|c|c|}
\hline Scatterer & Thickness $x, \mathrm{~cm}$ & $\rho x, g / \mathrm{cm}^{2}$ \\
\hline Aluminum & $7.62 \times 10^{-5}$ & $2.06 \times 10^{-4}$ \\
\hline Aluminized mylar & $5.08 \times 10^{-4}$ & \\
\hline Beryllium (vapor depoxited) & & $1.16 \times 10^{-3}$ \\
\hline Beryllium (rolled) & & $1.39 \times 10^{-3}$ \\
\hline Boron (on parylene $N$ ) & & $2.27 \times 10^{-3}$ \\
\hline Carbon (on parylene $\mathrm{N}$ ) & & 361. $\times 10^{-6}$ \\
\hline Carbon (on polyethylene) & & $\times 10^{-6}$ \\
\hline Mylar & $3.81 \times 10^{-4}$ & 533. $\times 10^{-6}$ \\
\hline Nickel & $1.0 \times 10^{-4}$ & $8.90 \times 10^{-4}$ \\
\hline Parylene C & $2.2 \times 10^{-4}$ & 283. $\times 10^{-6}$ \\
\hline Parylene $\mathbf{N}$ & $2.5 \times 10^{-4}$ & $\times 10^{-6}$ \\
\hline Parylene N (boron substrate) & $1.0 \times 10^{-4}$ & $\times 10^{-6}$ \\
\hline Parylene N (carbon cubstmate) & $8.5 \times 10^{-5}$ & $94.4 \times 10^{-6}$ \\
\hline Polyethylene & $3.54 \times 10^{-4}$ & 323. $\times 10^{-6}$ \\
\hline Titanium & $3.05 \times 10^{-4}$ & $1.38 \times 10^{-3}$ \\
\hline Saran & $1.02 \times 10^{-3}$ & \\
\hline
\end{tabular}




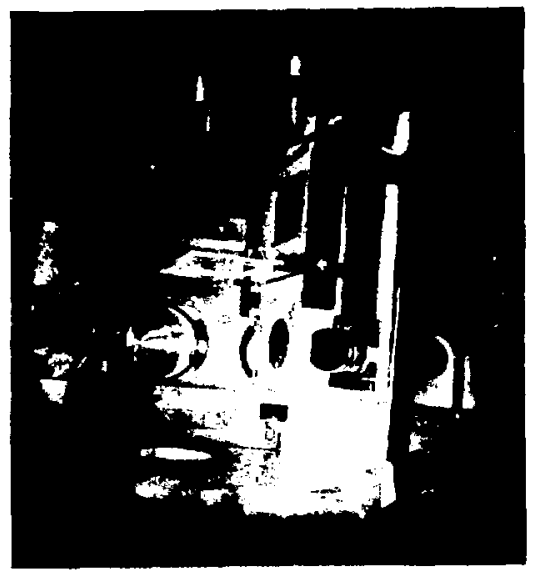

FIG. 2. Photographic view of scattering apparatus.

\section{PROCEDURE}

The scattering foil to be investigated was positioned in the evacuated scattering chamber as shown schematically in Fig. 1. The scattering foil was inclined at an angle of $45^{\circ}$ with respect to the incident beam on the horizontal plane and $90^{\circ}$ with respect to the incident beam on the vertical plane. The collimat or $C_{1}$ defined the size of the $x$-ray beam striking the scatterer and insured that the $x$-ray beam struck only the scatterer and not the mounting structure.

For the absolute scattering efficiency measurements using monoenergetic $x$ rays, detector No. 2 was kept at a position of $90^{\circ}$ with respect to the incident $x$-ray beam and looked at the front surface of the scattering foil through collimator $C_{3}$. The efficiency of this detector was known, having been measured for the energy range of 1 to $16 \mathrm{keV}$. Detector No. 1 was located on the axis of the $x$-ray beam about $8 \mathrm{~m}$ from the $x$-ray source, and when the foil was rotated up out of the beam this detector viewed the $\mathrm{x}$-ray source through collimator $C_{2}$.
The efficiency of this detector is alsa known for the energy range of 1 to $16 \mathrm{keV}$. The large distance to this detector and small collimator in front of it were necessary to reduce the $x$-ray flux to allow counting of the $x$-ray beam. The procedure was then to place the foil into the monoenergetic $x-r a y$ beam. and count the number of scattered $x$ rays as seen by detector No. 2 for a given time period, and at the same time record the data on a multichannel pulse height analyzer. The foil was then removed from the beam and the number of $\mathrm{x}$ rays as seen by detector No. I were counted for a fixed time as well as also being recorded on a multichannel pulse-height analyzer. By use of Eqs. (14) through (18), the absolute scattering efficiency as delined by Eq. (13) can be determined.

To measure the relative scattering efficiency of the foils, the experimental situp was nearly the same as described above with the exception that detector No. 2 was rotated $90^{\circ}$ to monitor the direct $x$-ray beam when the foil was removed from the beam. In addition, the $x$-ray beam used was a bremsstrahlung beam rather than a monoenergetic $x$-ray beam. When the incident bremsstrahlung beam was monitored with the foil out of the way and detector No. 2 positjoned on the beam axis, the current to the $x$-ray tube was reduced to enable the detector to count the incident flux and record the spectrum on the multichannel pulse-height analyzer. When the foil was in the beam, and detector No. 2 was rotated $90^{\circ}$ to measure the scattered beam and record the spectrum on the multichannel pulse-height analyzer, the current to the $x$-ray tube was increased to full value. By changing only the $x$-ray tube current and not the high voltage, the $x$-ray spectrum incident on the foil or detector was unchanged except for intensity. The scattered spectrum and the incident spectrum, both recorded on magnetic tape, were then processed by a computer rautine which converted the spectra from number of counts versus channel number to counts versus energy, and corrected them for detector efficiency. The corrected and converted scattered spectrum was then divided by the converted and corrected incident spectrum using a computer routine to provide the relative scattering efficiency versus $x$-ray energy for the foil. 


\section{RESULTS AND DISCUSSION}

\section{INCIDENT AND SCATTERED X-RAY SPECTRA}

The bremsstrahlung $x$-ray spectra from an aluminum anode and a copper anode are shown in Figs. 3 and 4. These spectra, corrected for detector efficiency, were recorded using $\mathrm{Si}(\mathrm{Li})$ detector No. 2 which had a $5.08 \times 10^{-3}$-cm-thick beryllium window. Most of the data obtained to determine the relative scattering efficiency was taken using the aluminum bremsstrahlung spectra incident on the various foils. The choice of the aluminum over the

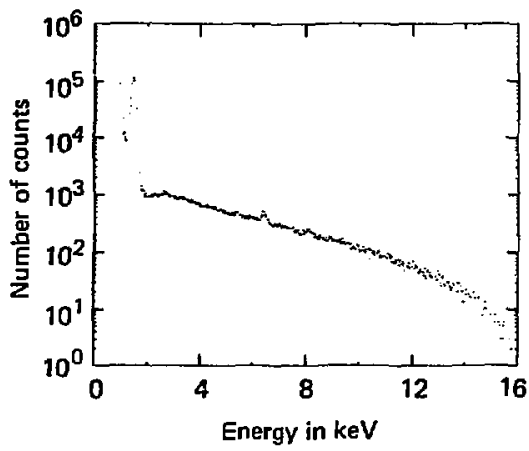

FIG. 3. Incident aluminum bremsstrahlung spectrum.

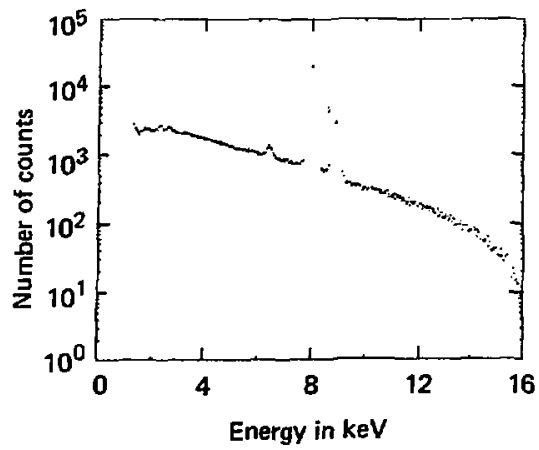

FIG. 4. Incident copper bremsstrahfung spectrum. copper spectra was to avoid having scattered fluorescent peaks with their accompanying escape peaks interfere with the Bragg peaks in the scattered spectra. A scattered peak due to the aluminum fluorescent peak did occur, but at least there was no escape peak present. Also, the resolution of the detectors allowed only one fluorescent peak for aluminum to occur, where two fluorescent peaks plus two escape peaks occurred for the scattered copper bremsstrahlung. In cases where the scattered aluminum peak interfered with the Bragg peaks seen in the scattered spectrum, the copper anode was also used to examine that region as it had no peaks in that energy region. Scattered spectra from a couple of the foils using both anodes are shown in Figs, 5-8. These spectra have been corrected for detector efficiency. Figures 5 and 6 are aluminum and copper bremsstrahlung spectra scattered from the rolled beryllium foil. The scattered fluorescent peaks, Bragg peaks, and escape peaks are identified. Figures 7 and 8 are the same bremsstrahlung spectra scattered from a thin aluminum foil. The aluminum foil shows a large fluorescent peak at $1.48 \mathrm{keV}$, which is much larger than the Bragg peaks and falls on top of the scattered aluminum fluorescent peak from the alun inum bremsstrahlung spectrum in Fig. 7 .

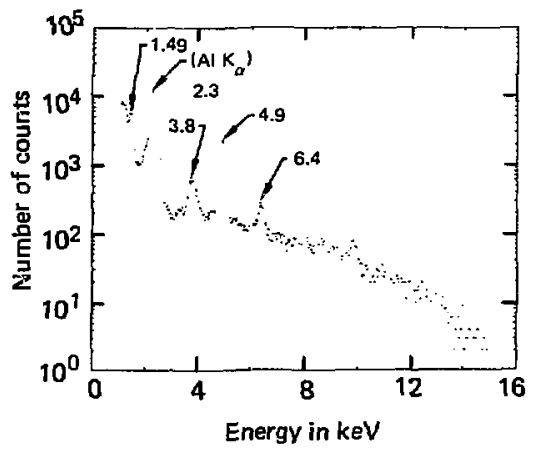

FIG. 5. Scattered aluminum hremsstrahlung spectrum from rolled beryllium foil. 


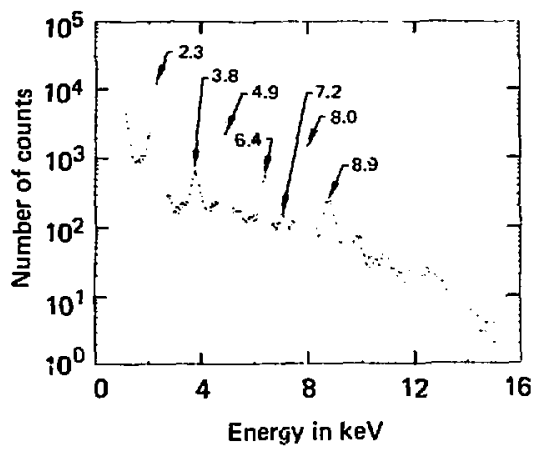

FIG. 6. Scattered copper bremsstrahlung spertrum from rolled beryllium foil.

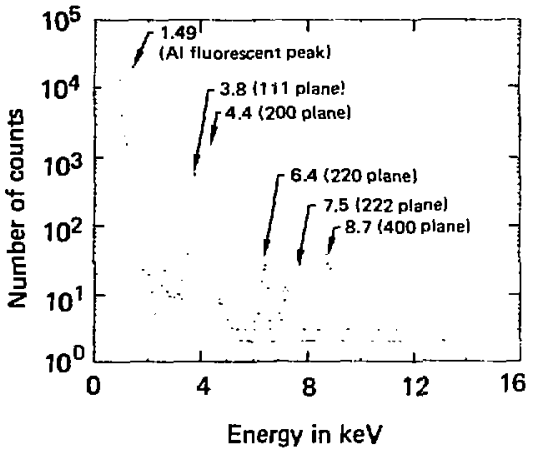

FIG. 7. Scattered aluminum bremsstrahlung spectrum from sluminum foil.

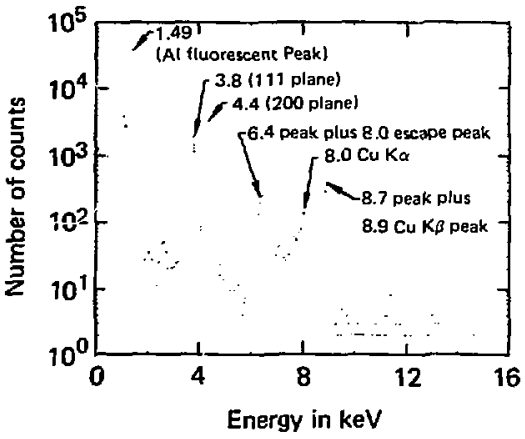

FIG. 8. Scattered copper bremsstrahlung spectrum from aluminum foil.

\section{RELATIVE SCATTERING EFFICIENCY}

The relative scattering efficiency of the foils listed in Table ! was determined using the proceciure described previously. The relative scattering efficiency versus incident $x$-ray energy for most of these scatterers is shown in Figs. 9-22. For some of the scatterers. fluorescent peaks as will as Biagg peaks are evident. The parylene- $C$ and saran reatterers both exhibit chlorine fluorescent peaks at approximately $2.6 \mathrm{keV}$ due to the chlorine in their composition. The aluminum, aluminized mylar. nickel, and ti.anium scatterers also exhibit nuorescent peaks at the appropriate $k$-shell energy for aluminum, nickel, and titanium.

The spectra shown in Figs. 9-22 are plotted out to $16 \mathrm{keV}$. However, since the incident bremsstrahlung spectra shown in Figs. 3 and 4 tuil off to zero at $16 \mathrm{keV}$, the statistics beyond 10 or $12 \mathrm{keV}$ are fairly poor. Likewise, at energies lower than approximately $1.5 \mathrm{keV}$ the data is not as reliable because the beryllium window on $\mathrm{Si}(\mathrm{Li})$ detector No. 2 cuts off the low energy $x$ rays, and the electronic noise of nir sounting system becomes a more predominant effect.

\section{ABSOLUTE SCATTERING EFFICIENCY}

The absolute scattering efficiency was determined for four of the scatterers. These were the two beryllium foils, the carbon on parylene foil, and the boron on parylene foil. The procedure as described previously is very time consuming since some of the count rates as measured by $\mathrm{Si}(\mathrm{Li})$ detector No. 2 were very low, and the time required to accumulate a good spectrum exceeded 6 hours for a single energy point. Since eight energy points in the region from 1.5 to $8 \mathrm{keV}$ were used for each scattering efficiency measurement, the absolute scattering efficiency was measured for only four foils. Figures 23-26 show the measured scattering efficiency as well as the calculated scattering efficiency as described previously. In addition the relative scattering efficiency normalized at one energy point is also shown. The normalization was done at an energy point which was not affected or influenced by a Bragg peak. After normalization. the agreement between the relative scattering efficiency eurves and the measured absolute scattering efficiency at distinct energy points is remarkably 


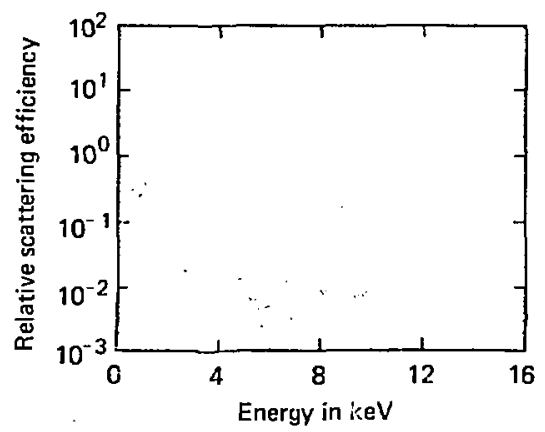

:IG. 9. Relative scattering efficicucy for aluminum.

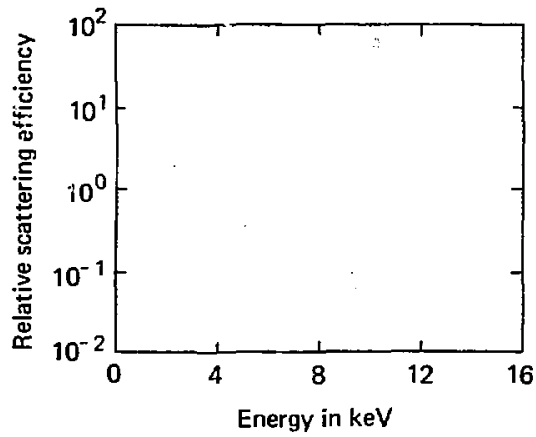

FIG. 10. Relative scattering efficiency for aluminized mylar.

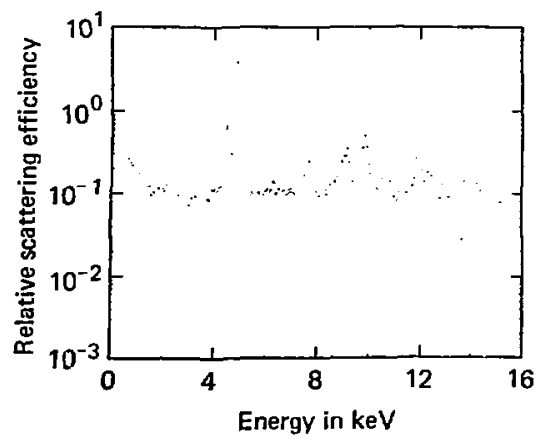

FIG. 11. Relative scattering efficiency ro plated beryllium.

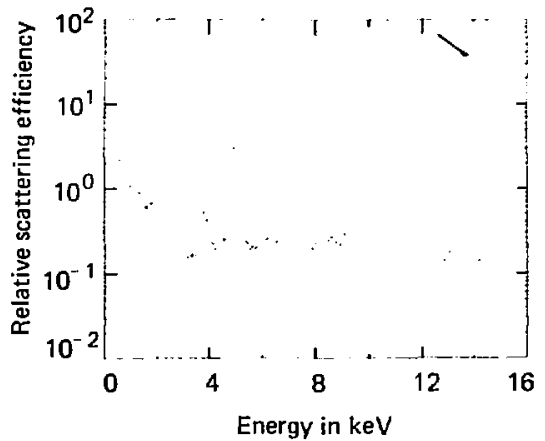

FIC. 12. Retative scattering efficiency for rolled berylliun.

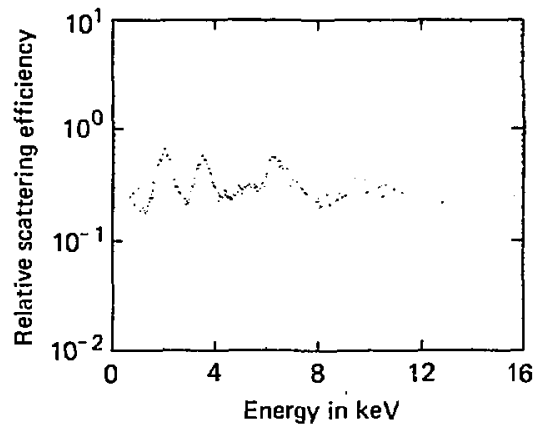

FIG. 13. Relative scattering efficiency fot boton on parylene.

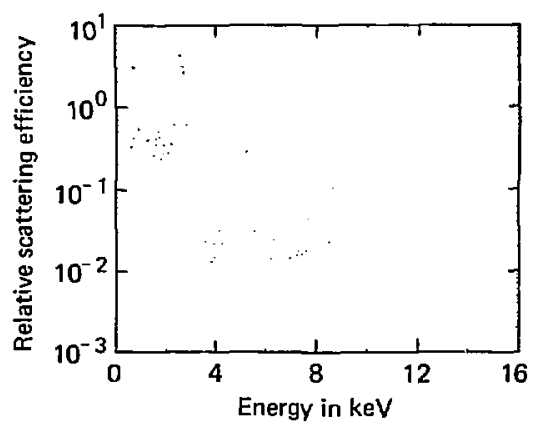

FIG. 14. Relative scattering efficiency for carbon on parylene. 


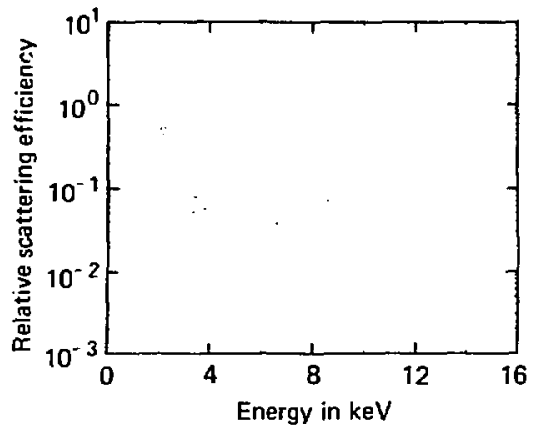

FIG. 15. Relative scattering efficiency for carbon on polyethylene.

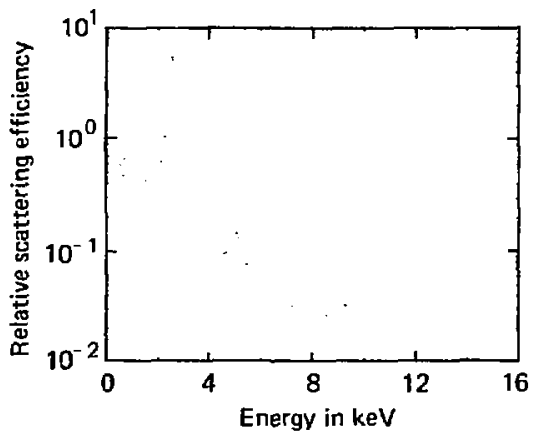

FIG. 16. Rejative scattering efficiency for mylar.

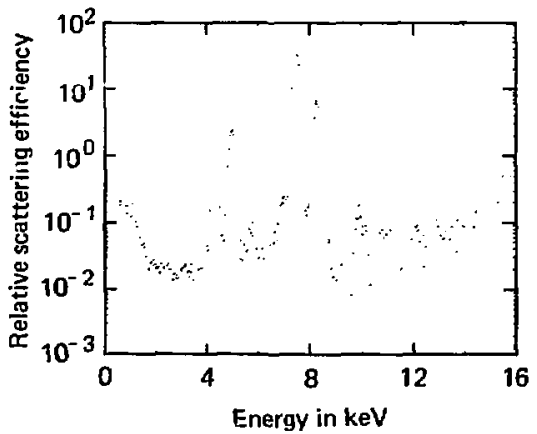

FIG. 17. Relative scattering efficiency for nickel.

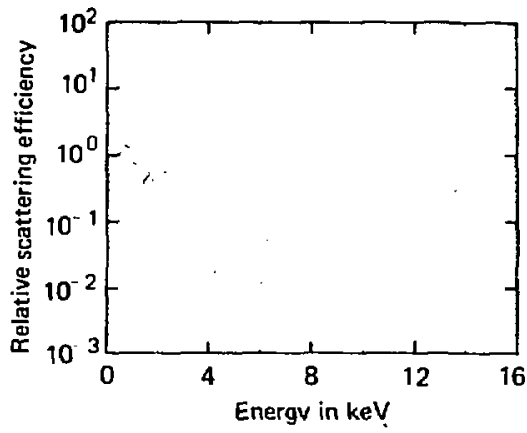

FIC. 18. Relative scattering efficiency for parylene C.

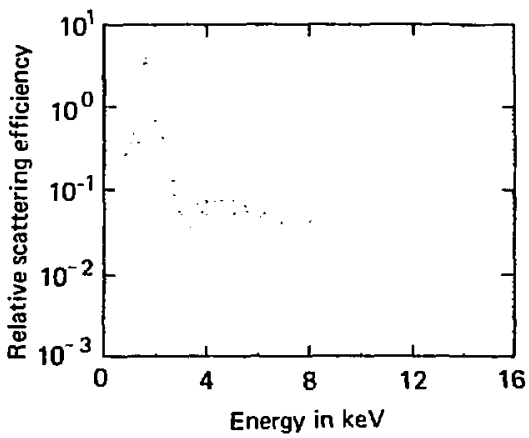

FIG. 19. Relative scattering efficiency for parylene N.

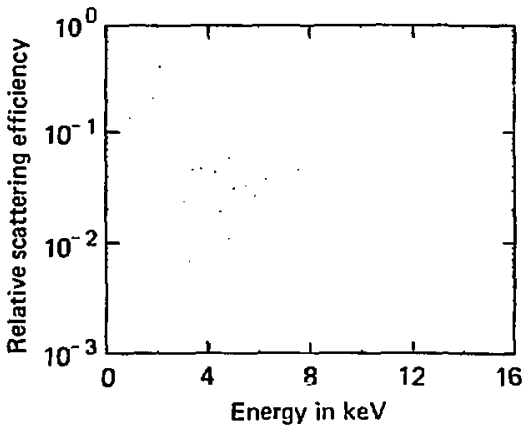

FIG. 20. Relative scattering efficiency for polyethylene. 


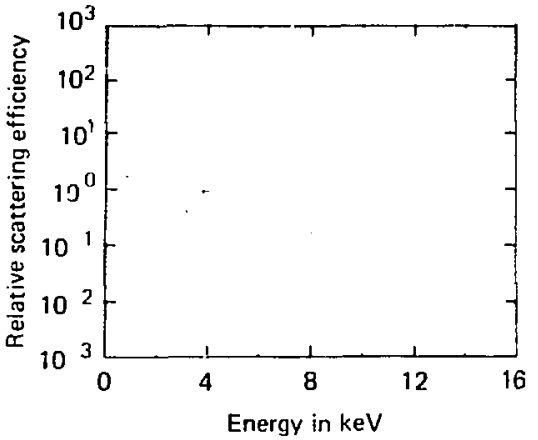

FIC: 21. Relative scattering efficiency for titanium.

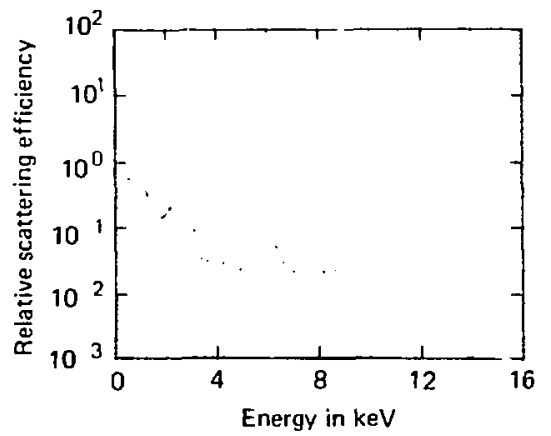

F1(. 22. Relative scaltering efficiency for saran.

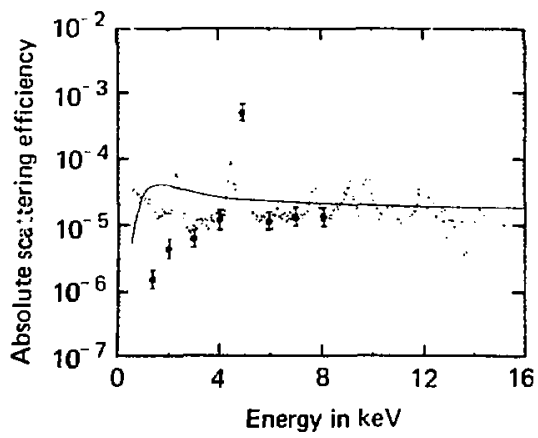

FIG. 23. Absolute scattering efliciency for plated beryllium.

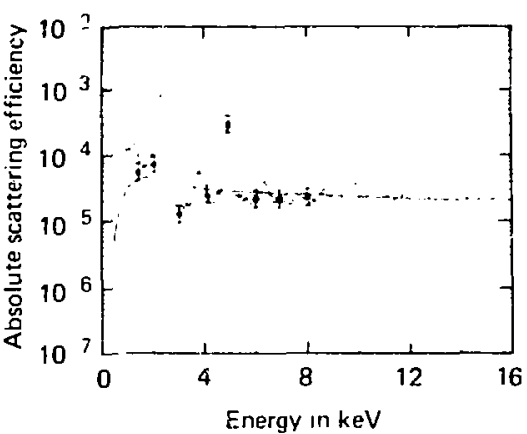

FIC. 24. Ahsolute seattering efficiency for rotled beryllium.

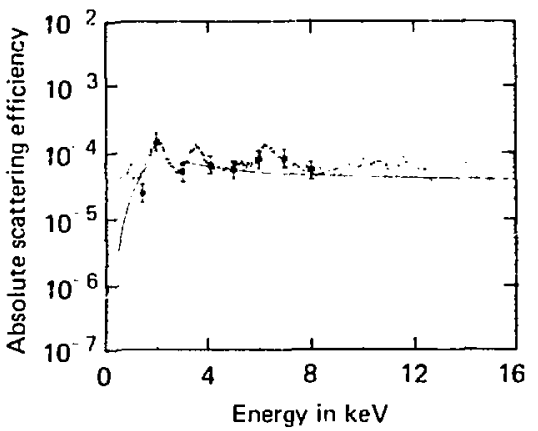

FIC. 25. Absolute scattering efficiency for boron on parylene.

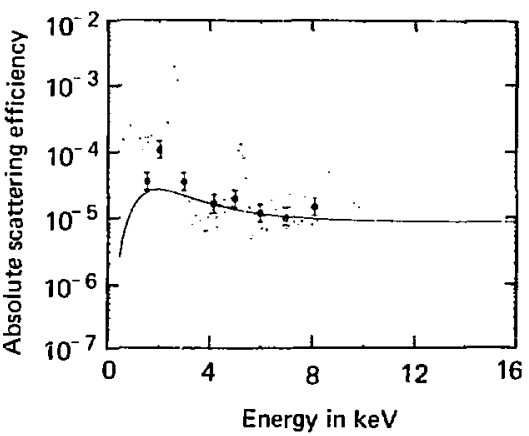

FIG. 26. Absolute scattering efficiency for carbon on parylene. 
food The brackets on the calitration pounts at dis. unct energy pounts represent an estimated uncertannty in the measurements of approximiately $25 \%$. Thes is due frimaroly to a small uncertainty involved in detcrmining the number of $x$ rays in the incident nuoteseent peak and a much larger uncertainty in. volved in delermining the nuniher of $x$ rays in the scattered fluorescent peak. Since the incident filtered fluorescent $x$-riy spectra is nor 100 y monoenergetic but only $89-98 \%$ spectrally pure, a small amount of contaminant that happens to the Bragg scattered can become a much more signifi. tunt feature of the scattered spectrum than the scas! tesed tiuorescent peak. Due to the $185 \mathrm{eV}$ resolution of the sill i) detector. these Bragg-seattered contaminant peaks often partially overlap the scattered nuorescent peaks. naking it difficult to determine their magnitude.

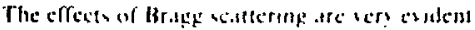

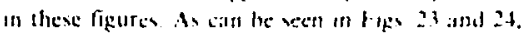
the Brage scitleteng peith irom ian differem puces of hergllium are different. The meht he expested since they were fatiricated differemh and suce the orientation of the trystal platres a as hot convidered when they were mournted an their hellers. B3 reorentung the forls a thinge on the Brage satternge meaks could be aceomplshed. The calculitted sialtering effictency, which does mot molude br:teg saitstering. aprees with the measurements fatrly well for

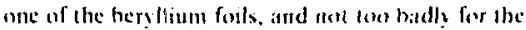

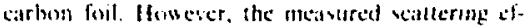
ficiency for the vaporsplated ierglhiom soll. although in agreemen " ith the calculation an higber energies, disagress with the calculaten considerably at the lower energies $(1-i \mathrm{keV})$.

\section{CONCLUSIONS}

All of the scatterers examined in this experjment exhibited Bragg-like $x$-ray diffraction. The number of diffraction peaks was generally grealer for the metallic seatterers thin for the others. As was mentioned previously, some of the scalterers also produced fluorescent $x$-ray peaks in the energy region of interest when $x$ rays were incident on them. Since the present calculational model for scilltering efficiency does not account for these effects, it only gives an approximate value when compared to the measured scattering efficiency. If the scatterers were truly amorphous. the anreement should be quite good with the exception of possible fuorescent peaks.

Since even the sime types of scatterers maly have different erystalline-like orientatioss, the only valid method of determining the scattering of ficiency of a particular scattering foil is to experimentally measure it. 


\section{REFERENCES}

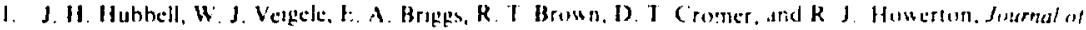
Phascal ond Chemeal Referinge Data 4, 471 (19? S)

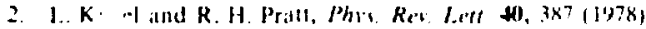

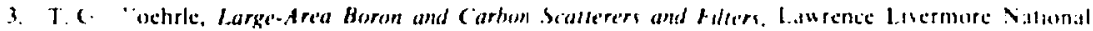

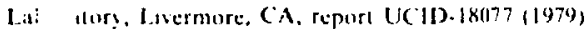

4. J. L. Ciants, Neal. Inst and Me'hest 102. 7 11972,

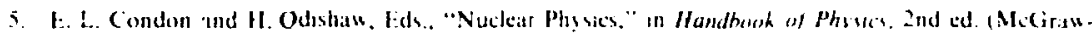
Hill, Neu York, 1967), Pist 9. pp. 116-119

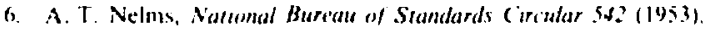

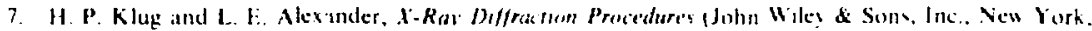
195.4) p. 122.

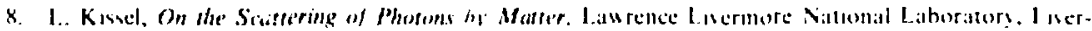
more, $C A$, imernal report XRM-78-125 (1978)

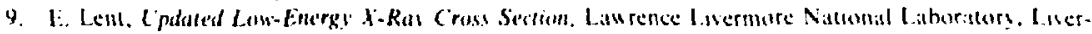
mure, CA. internal report DAG.77.22 (1977)

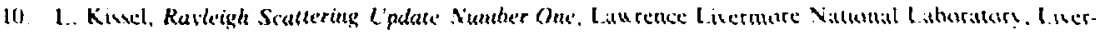
more. CA. internal report XRMi-79)-7 (1979).

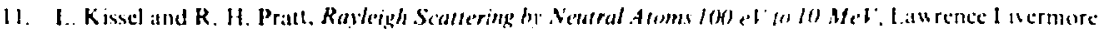
Nistional Laboratory, Livermore, CA, report UCRL-82507 (19)79).

12. I. Giaines, IEEE Transations on Nuclear Science NS-26, 1420 (1978).

3. J. Hetcher, Livermone-Time-Sharing Sistem, Lawrence livermare National laboratory. L.sermere. CA, report I.TSS-6 (1970). 